\title{
Virtual Makeover Software
}

\author{
Asma Sajjad \\ Department of \\ Computer Science \\ Faculty of Basic and \\ Applied Sciences \\ International Islamic \\ University Islamabad
}

\author{
Sadia Arshad \\ Department of \\ Computer Science, \\ Faculty of Basic and \\ Applied Sciences \\ International Islamic \\ University Islamabad
}

\author{
Afsheen Tariq \\ Department of \\ Computer Science \\ Faculty of Basic and \\ Applied Sciences \\ International Islamic \\ University Islamabad
}

\author{
Ayesha Shoaib \\ Department of \\ Computer Science \\ Faculty of Basic and \\ Applied Sciences \\ International Islamic \\ University Islamabad
}

\begin{abstract}
Many face makeover software provide the facility of doing makeup either by using free hand drawing or using templates in which, points can be adjusted according to face and then application of make-up is performed, which often does not provide high-quality output. Automatic detection of facial features, such as eyes, lips and face line etc, seems to be easier and convenient way of applying make-up but there is no software that provides automated facial features detection technique. This application provides this facility by using image processing techniques which can automatically detects hair line, eyes and lips to apply make-up.
\end{abstract}

\section{Keywords}

Image Processing, Feature Extraction, Eye Localization, Eye Detection, Lip Localization, Lip Detection.

\section{INTRODUCTION}

In this application user can upload his/her picture and can easily apply different hairstyles, hats and frames which are stored in the database as well as user will have the facility to apply eye shades, lipsticks, blush and concealer. Furthermore, it also provides options through which user can move up, down, left, right to adjust hairstyle and can increase or decrease its width and height for best setting on his/her picture. User can also adjust eye shadow, blush and concealer. It offers "gamma correction" to adjust the brightness of an image. In this application user is also enabled to apply different effects on image i.e. grayscale, sepia, jitter and invert color. And options like reloading image, save print, clear undo, redo and results of the makeover effect can also be shown by viewing images of before and after editing.

\subsection{Upload Picture}

This module is for uploading the picture of the user. The user can upload any picture of format BMP, JPEG or PNG because they support true color format.

\subsection{Selecting Hairstyle}

User can select long, medium or short hairstyles of four types of shades black, blonde, brown and red. The given hairstyles are in PNG format. We have opted for PNG because JPEG and GIF do not support transparency. The hairstyles have their background transparent, so that when it is inserted the user can only view the hairstyle being inserted.

\subsection{Selecting Eye and Lipstick shades}

A wide variety of eye and lipstick shades has been provided to suit the user needs. The color dialogue box will provide user wide range of shading options, which user can opt with respect to his/her requirements.

\subsection{Accessories Insertion}

The application provides more than 20 hats and over 10 photo frames option which can be used at user's will.

\section{PREVIOUS WORK}

There are applications available which provide makeover functionality on CD's and online. These applications do not use any automatic detection. Entire work is performed manually. User either has to drag and drop templates that have been provided for face, eyes and lips or user is required to draw lines around lips and eyes which is totally ineffective.

\section{PROPOSED METHODOLOGY \\ 3.1.Major Modules}

Our system consists of following modules that are also the building blocks of many face recognition applications:

\subsubsection{Loading Image}

Image with proper lightning effect is loaded.

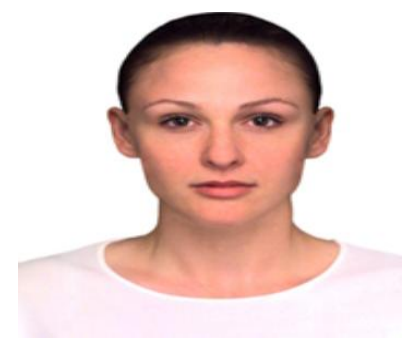

Figure 1: Uploaded Picture

\subsubsection{Face Localization}

The purpose of face localization is spotting whether or not face is present, also returning its ultimate location. Image segmentation is applied to check whether face is present or not[1]. It basically applies HSL (Hue Saturation and Luminance) color space to recognize the skinny area and fill rest of the area with black color. The limitation lies here is that background of an image can only be white otherwise hue will not be calculated properly which may affect the results of an image [2].

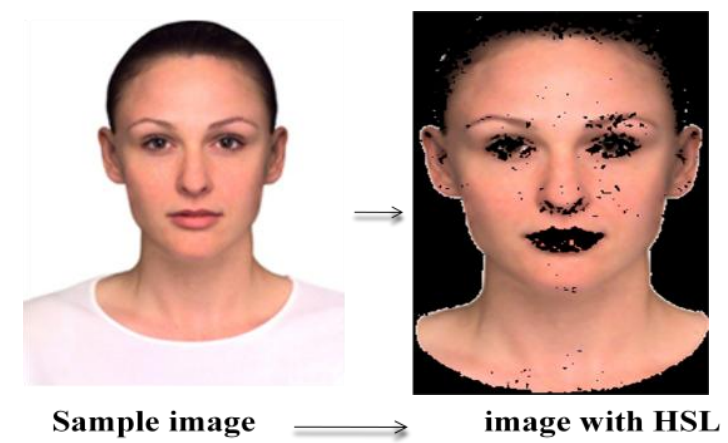

Figure 2: Image with Hue 


\subsubsection{Face Segmentation}

In the HSL color space the hue value for the skin is determined [2]. All the things except skin are converted to background. Then as system has to recognize hairline so different filters are applied to detect hairline from an image. Gray scaling, Gaussian filter, opening, sharpening, threshold, dilation and in the end sobel edge detector are applied through which hairline is detected [3].
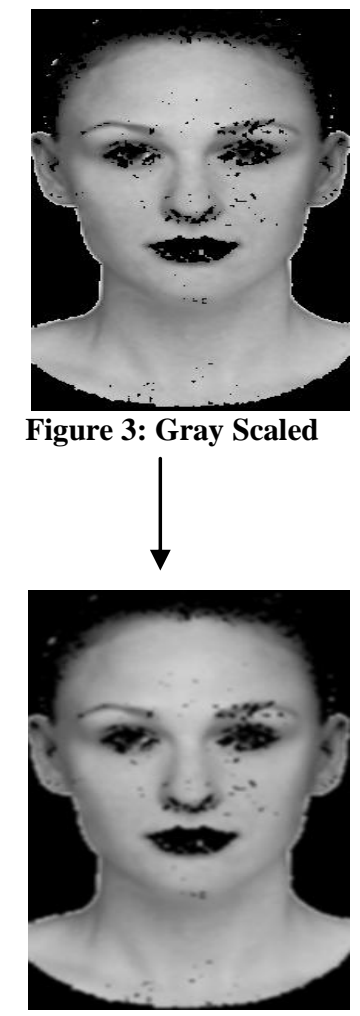

Figure 4: Guassian Blur

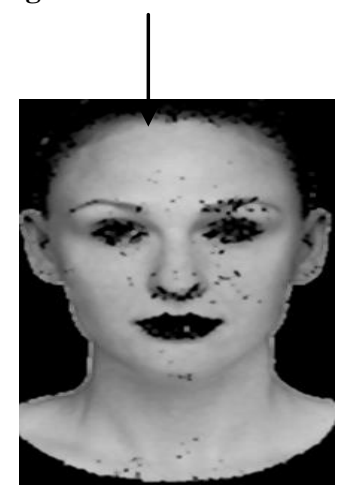

Figure 5: Sharpen
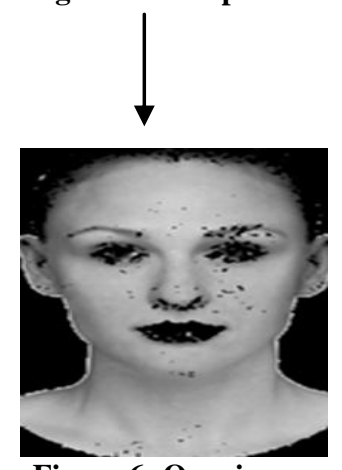

Figure 6: Opening

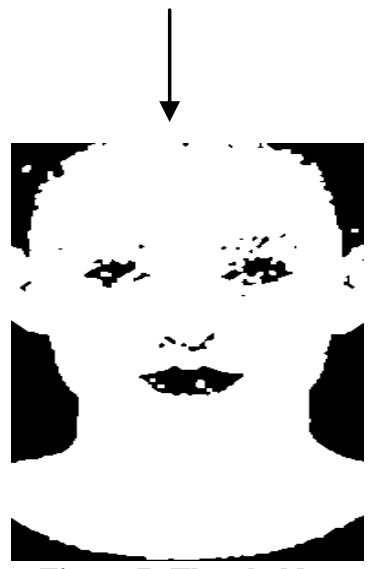

Figure 7: Threshold
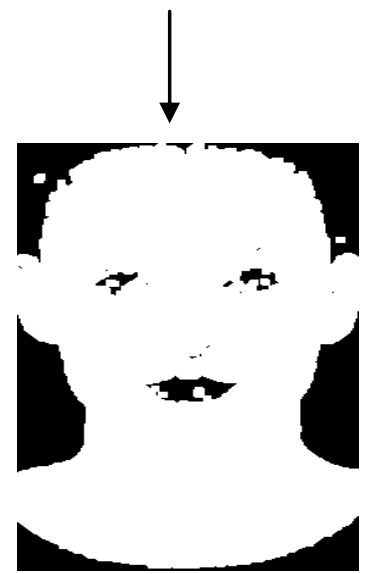

Figure 8: Dilation
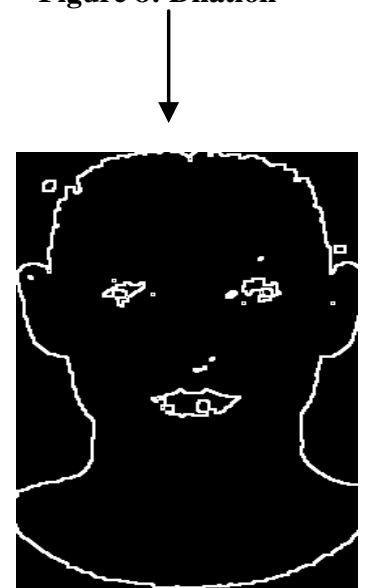

Figure 9: Sobel Edge Detector

\subsubsection{Face Zone Division}

When the image will be normalized then it will be divided into zones. Line will be drawn on major axis (y-axis) and minor axis (x-axis). Purpose of major and minor axis is to identify the skinny area. On the basis of this division, further processing will be done. i.e. feature extraction. 


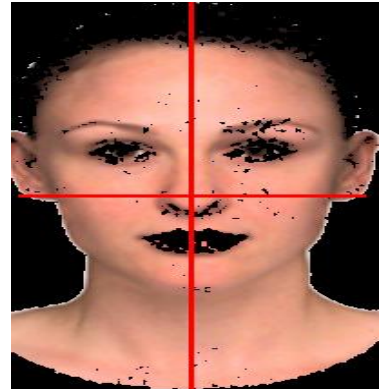

Figure 10: Face division into zones

\subsection{Feature Extraction}

We have to extract features to apply makeup on eyes and lips.

\subsubsection{Eye Localization \& Detection}

Eye region is first of all localized for eye detection. Eyes are localized on the basis of hue which does not belong to the skin region. Rectangles are created on the basis of hue as eyes are non skinny area so it is black from eye area. Then left and right eye rectangle will be created. Both eyes are cropped. Filters are applied to remove small objects and to identify eye area properly.

Image is converted into grayscale then edge detectors will be applied. Morphological operations are applied for smoothing and to remove small objects and finally fill holes are applied to fill eye area properly[4].
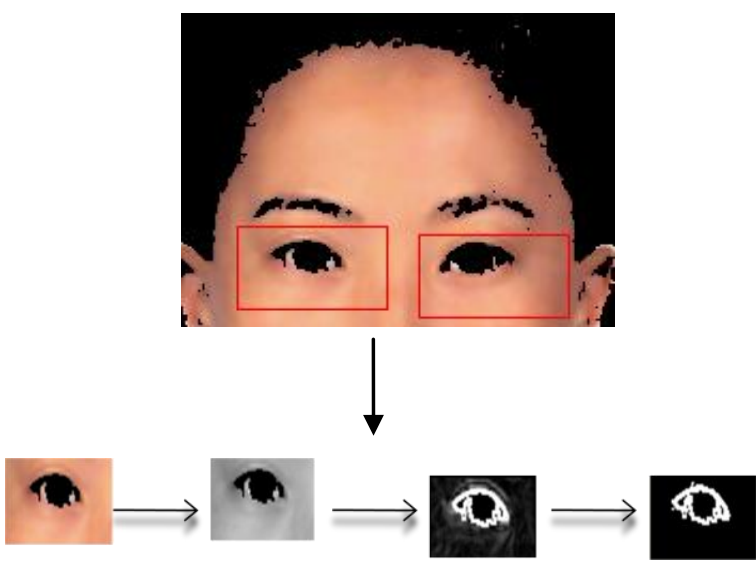

(a)

(b)

(c)

(d)
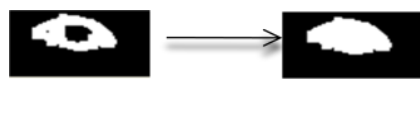

(f)

(g)

Figure 11: Eye Localization and detection (a)Cropping Eyes (b) Grayscale (c) Sobel Edge Detector (d) Threshold (e) Dilation(f) Fill Holes (g) Erosion

\subsubsection{Lip Localization \& Detection}

On the basis of major and minor axis which has been calculated, area below minor axis is identified. The non skinny area below minor axis is only lips. Rectangle is created on that non skinny area and then it will be cropped. To remove small objects different filters are applied.

Image is converted into grayscale then edge detectors, morphological operations and fill holes are applied for proper detection of lips[5].
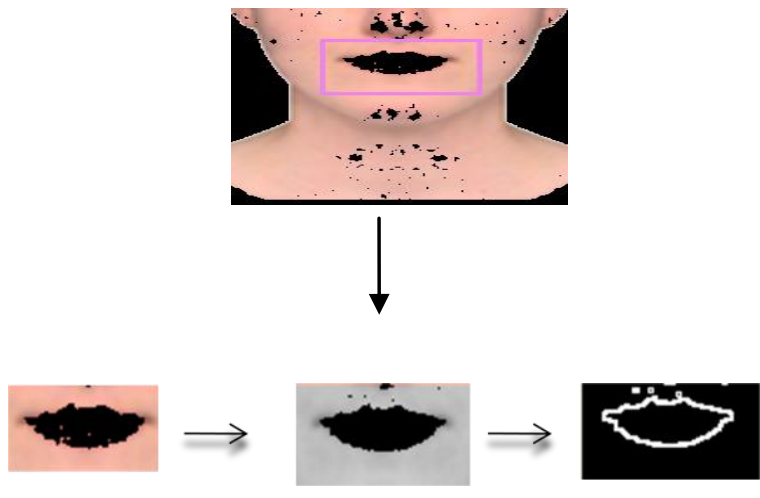

(a)

(b)

(c)

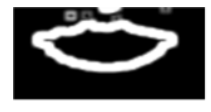

(d)

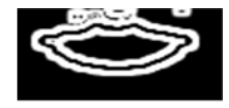

(e)

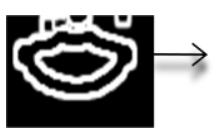

(f)

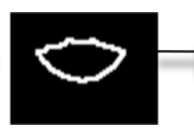

(g)

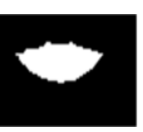

(h)

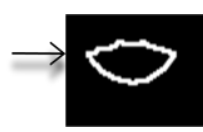

(I)
Figure 12: Lip localization and detection (a)Cropping Lips (b) Gray scale (c) Threshold (d) Guassian Blur (e) Sobel Edge Detector (f) Dilation (g) Erosion (h) Fill holes (I) Sobel Edge Detector

\section{CONSTRAINTS}

The proposed methodology gives best results when few constraints are followed. The picture should be taken on a white background. It should have the full face exposed i.e. complete forehead, ears and chin area. The hair should be tightly tucked back.

\section{RESULTS}

The experimental results shows that it gives more than $80 \%$ accuracy.

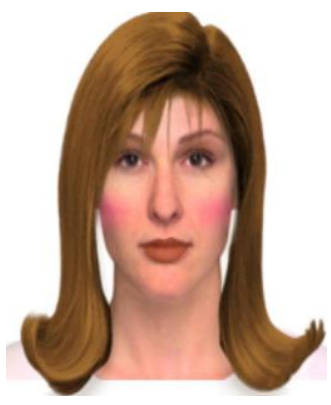

Figure 13: Linda with her new look 


\section{CONCLUSION}

Through the usage of this software the user will be able to choose one of the most suitable hairstyle and makeup.

It facilitates the user in selecting the hairstyle, makeup and accessories. This software will prove to be very popular commercial product. It will be utilized by saloons for acquiring their customer requirements and also by individuals personally to let their stylist know exactly what kind of makeover they want.

\section{FUTURE ENHANCEMENTS}

The introduction of computer applications in the field of beauty is not very old. There is lot of scope for adding new very strong and most wanted features in this application.

The software can be improved by adding the facility for male's beard and moustache. More shades of hairstyles can be added i.e. burgundy, ash, grey, etc. $3 \mathrm{D}$ view i.e. the user can view his/her picture from any angle. User can do the dress makeover. Automated detection of best makeover for users face.

\section{REFERENCES}

[1] Irosog. Face Detection in in C\#. Code Project.com, 21 Feb 2012.

$$
6
$$$$
\text { Sept }
$$

<http://www.codeproject.com/Articles/39060/Face-

Detection-in-C>
[2] Cui, F.-y., Li-jun Zou ; Bei Song, Edge feature extraction based on digital image processing techniques, Proceedings of the IEEE International Conference on Automation and Logistics, Qingdao, China Sept2008. 29 Sept 2012

<http://ieeexplore.ieee.org/xpl/login.jsp?tp=\&arnumber=4636 554\&url=http\%3A\%2F\%2Fieexplore.ieee.org\%2Fxpls \%2Fabs_all.jsp\%3Farnumber\%3D4636554>

[3] R. Fisher, S. Perkins, A. Walker and E. Wolfart.Image Processing Learning Resources, Copyright (C) 2000 Robert Fisher, Simon Perkins, Ashley Walker and Erik $\begin{array}{llll}\text { Wolfart } & \text {. } & 29 & \text { Sept }\end{array}$ <http://homepages.inf.ed.ac.uk/rbf/HIPR2/dilate.htm>

[4] Iregen Khuedchenia. Introduction to morphology Operation on Images. Computer Vision Talk, 16 Feb 2011. 29 Sept 2012 <http://computer-visiontalks.com/2011/02/introduction-to-morphologyoperations-on-images/>

[5]Sohail Safdar. Matlab Code for Dialation and Erosion. Chillmania Blog, 17 June 2010. 5 October 2012. <http://chilmania.blogspot.com/2010/06/matlab-codefor-dilation-and-erosion.html > 